University of Wollongong

Research Online

Faculty of Science, Medicine and Health -

Papers: Part B

Faculty of Science, Medicine and Health

$1-1-2014$

\title{
Identification of the linkage group of the $Z$ sex chromosomes of the sand lizard (Lacerta agilis, Lacertidae) and elucidation of karyotype evolution in lacertid lizards
}

\author{
Kornsorn Srikulnath \\ Nagoya University, Kasetsart University \\ Kazumi Matsubara \\ University of Canberra \\ Yoshinobu Uno \\ Nagoya University \\ Chizuko Nishida \\ Hokkaido University \\ Mats M. Olsson \\ University of Sydney, molsson@uow.edu.au
}

See next page for additional authors

Follow this and additional works at: https://ro.uow.edu.au/smhpapers1

Publication Details Citation

Srikulnath, K., Matsubara, K., Uno, Y., Nishida, C., Olsson, M. M., \& Matsuda, Y. (2014). Identification of the linkage group of the $Z$ sex chromosomes of the sand lizard (Lacerta agilis, Lacertidae) and elucidation of karyotype evolution in lacertid lizards. Faculty of Science, Medicine and Health - Papers: Part B. Retrieved from https://ro.uow.edu.au/smhpapers1/993

Research Online is the open access institutional repository for the University of Wollongong. For further information contact the UOW Library: research-pubs@uow.edu.au 


\title{
Identification of the linkage group of the $Z$ sex chromosomes of the sand lizard (Lacerta agilis, Lacertidae) and elucidation of karyotype evolution in lacertid lizards
}

\author{
Abstract \\ The sand lizard (Lacerta agilis, Lacertidae) has a chromosome number of $2 n=38$, with 17 pairs of \\ acrocentric chromosomes, one pair of microchromosomes, a large acrocentric $Z$ chromosome, and a \\ micro-W chromosome. To investigate the process of karyotype evolution in L. agilis, we performed \\ chromosome banding and fluorescent in situ hybridization for gene mapping and constructed a \\ cytogenetic map with 86 functional genes. Chromosome banding revealed that the $Z$ chromosome is the \\ fifth largest chromosome. The cytogenetic map revealed homology of the L. agilis $Z$ chromosome with \\ chicken chromosomes 6 and 9. Comparison of the L. agilis cytogenetic map with those of four Toxicofera \\ species with many microchromosomes (Elaphe quadrivirgata, Varanus salvator macromaculatus, \\ Leiolepis reevesii rubritaeniata, and Anolis carolinensis) showed highly conserved linkage homology of $L$. \\ agilis chromosomes (LAG) 1, 2, 3, 4, 5(Z), 7, 8, 9, and 10 with macrochromosomes and/or \\ macrochromosome segments of the four Toxicofera species. Most of the genes located on the \\ microchromosomes of Toxicofera were localized to LAG6, small acrocentric chromosomes (LAG11-18), \\ and a microchromosome (LAG19) in L. agilis. These results suggest that the L. agilis karyotype resulted \\ from frequent fusions of microchromosomes, which occurred in the ancestral karyotype of Toxicofera \\ and led to the disappearance of microchromosomes and the appearance of many small \\ macrochromosomes.

\section{Publication Details} \\ Srikulnath, K., Matsubara, K., Uno, Y., Nishida, C., Olsson, M. \& Matsuda, Y. (2014). Identification of the \\ linkage group of the $Z$ sex chromosomes of the sand lizard (Lacerta agilis, Lacertidae) and elucidation of \\ karyotype evolution in lacertid lizards. Chromosoma: biology of the nucleus, 123 (6), 563-575.
}

\section{Authors}

Kornsorn Srikulnath, Kazumi Matsubara, Yoshinobu Uno, Chizuko Nishida, Mats M. Olsson, and Yoichi Matsuda 


\title{
Identification of the linkage group of the $\mathrm{Z}$ sex chromosomes of the sand lizard (Lacerta agilis, Lacertidae) and elucidation of karyotype evolution in lacertid lizards
}

\author{
Kornsorn Srikulnath \& Kazumi Matsubara \& \\ Yoshinobu Uno \& Chizuko Nishida \& Mats Olsson \& \\ Yoichi Matsuda
}

\begin{abstract}
The sand lizard (Lacerta agilis, Lacertidae) has a chromosome number of $2 \mathrm{n}=38$, with 17 pairs of acro- centric chromosomes, one pair of microchromosomes, a large acrocentric $\mathrm{Z}$ chromosome, and a micro-W chromo- some. To investigate the process of karyotype evolution in

L. agilis, we performed chromosome banding and fluores- cent in situ hybridization for gene mapping and construct- ed a cytogenetic map with 86 functional genes. Chromosome banding revealed that the $\mathrm{Z}$ chromosome is the fifth largest chromosome. The cytogenetic map re- vealed homology of the L. agilis $\mathrm{Z}$ chromosome with chicken chromosomes 6 and 9. Comparison of the L. agilis cytogenetic map with those of four Toxicofera species with many microchromosomes (Elaphe quadrivirgata, Varanus salvator macromaculatus, Leiolepis reevesii rubritaeniata, and Anolis carolinensis) showed highly conserved linkage homology of L. agilis chromosomes (LAG) 1, 2, 3, 4, 5(Z), 7, 8, 9 , and 10 with macrochromosomes and/or macrochromosome segments of the four Toxicofera species. Most of the genes located on the microchromosomes of Toxicofera were localized to LAG6, small acrocentric chromosomes (LAG11-18), and a microchromosome (LAG19) in L. agilis. These results suggest that the L. agilis karyotype resulted from frequent fusions of microchromosomes, which occurred in the an- cestral karyotype of Toxicofera and led to the disappear- ance of microchromosomes and the appearance of many small macrochromosomes.
\end{abstract}

Introduction

Comparison of the draft genome assembly of the green anole (Anolis carolinensis) with the genome sequence of chicken (Gallus gallus) suggested a high level of conservation of the genetic linkages between the two species (ICGSC 2004; Alföldi et al. 2011). This genome information provided a new perspective on the comparative genomics of Reptilia and Aves, which in turn facilitated extensive comparison of genomic structures between the two taxa at the molecular level. Chromosome maps that compared each of the Chinese soft-shelled turtle (Pelodiscus sinensis), Siamese crocodile (Crocodylus siamensis), water monitor lizard (Varanus salvator macromaculatus), Japanese four-striped rat snake (Elaphe quadrivirgata), and butterfly lizard (Leiolepis reevesii rubritaeniata) with the chicken revealed a high level of conservation of chromosomes among the members of Aves, Testudines, Crocodylia, and Squamata (Matsuda et al. 2005; Matsubara et al. 2006, 2012; Srikulnath et al. 2009b, 2013; Uno et al. 2012) since the time when the Sauropsida (all existing reptiles and birds) diverged from the Synapsida around 320 million years ago (MYA), based on the dating of Shedlock and Edwards (2009).

Reptiles display considerable diversity in their sex chro- mosomes and the systems they use for sex determination. Whereas all crocodilians, the tuatara, most turtles, and some lizards have temperature-dependent systems that determine sex (Head et al. 1987; Lang and Andrews 1994; Cree et al. 1995; Ciofi and Swingland 1997), almost all snakes (Serpentes) exhibit genotypic sex determination (GSD); whereas most snakes have morphologically differentiated ZZ/ZW-type sex chromosomes, and many lizards and some turtles exhibit GSD with both male and female heterogamety $\left(\mathrm{XY}, \mathrm{X}_{1} \mathrm{X}_{2} \mathrm{Y}, \mathrm{ZW}\right.$, and $\mathrm{Z}_{1} \mathrm{Z}_{2} \mathrm{~W}$ ) (Olmo and Signorino 2005). However, the origin of the sex chromosomes in reptiles and the processes by which they differentiated are poorly under- stood owing to the limited number of species for which sex- linked genes have been identified. Cytogenetic maps that compare the chicken with each of two turtle species, the black marsh turtle (Siebenrockiella crassicollis) and P. sinensis, in- dicated that the S. crassicollis X chromosome corresponds to chicken chromosome 5 and that the P. sinensis Z sex chromo- some corresponds to chicken chromosome 15 (Kawagoshi et al. 2009, 2012). In squamate reptiles, such as snakes, the origins of the sex chromosomes differ from those of the chicken $Z$ chromosome, although snake sex chromosomes use the ZZ/ZW system found in birds (Matsubara et al. 2006 , 2012 ). A cytogenetic map that compared E. quadrivirgata (Serpentes) with chicken revealed that, whereas the chickenZ-linked genes were localized to the short arm of snake chromosome 2, the chicken homologs of the snake Z-linked genes were 
localized to chicken chromosomes 2 and 27 (Matsubara et al. 2006, 2012). The micro-X chromosome of $A$. carolinensis (Iguania) is homologous to chicken chromosome 15, which corresponds to the P. sinensis Z chromosome (Alföldi et al. 2011). Moreover, the $\mathrm{Z}$ chro- mosome of the Hokou gecko (Gekko hokouensis, Gekkota) has homology with the chicken $\mathrm{Z}$ chromosome (Kawai et al. 2009). These results collectively suggest that independent differentiation of sex chromosomes in each lineage of reptiles resulted in them using different systems for the genetic deter- mination of sex.

Compared with avian and testudine species, the karyotypes of squamate species are relatively diverse, in terms of both their chromosome number $(2 \mathrm{n}=30-50)$ and theirchromosome morphology (Olmo and Signorino 2005). In squamate reptiles, macro and microchromosomes are commonly found in the Dibamidae, Scincoidea, and Episquamata (Toxicofera and Lacertoidea), excluding the Lacertidae (Olmo and Signorino 2005; Pyron et al. 2013). In our previous studies (Srikulnath et al. 2009b, 2013), we compared the chromosomal locations of 86 functional genes among three Toxicofera species $[E$. quadrivirgata (Serpentes), V. salvator macromaculatus (Anguimorpha), and L. reevesii rubritaeniata (Iguania)] (Vidal and Hedges 2005; Pyron et al. 2013) and revealed that most macrochromosomes were homologous. Nonetheless, these studies revealed several chromosomal rearrangements among the species. The rearrangements included centric fission/fusion of macrochromosomes, telomere-to-telomere tandem fusion between macrochromosomes and between macro and microchromosomes, and centromere repositioning. By contrast, few or no microchromosomes are found in the Lacertidae of Lacertoidea and Gekkota. The observation that the karyotype of Lacertidae (lacertid lizards) usually has only one pair of microchromosomes (Odierna et al. 1993; Olmo and Signorino 2005 ) l eads us to suppose that microchromosomes in this lineage disappeared as a conse- quence of fusions between macro and microchromosomes and/or between microchromosomes. However, there have been no reports that confirm this. Comparative gene mapping between lacertid lizards and other squamate reptiles of the Toxicoferaclade is required to clarify the process of karyotype evolution, including the dynamic reorganization of microchromosomes in Squamata.

Lacertid lizards (Lacertidae, Lacertoidea), which comprise 40 genera with 318 species (Uetz 2014), inhabit Africa and almost the whole of Eurasia. The diploid chromosome number of most lacertid lizards is 38; this comprises 10 pairs of large acrocentric chromosomes, eight pairs of small acrocentric chromosomes, and one pair of microchromosomes (Olmo and Signorino 2005). Most lacertid lizards exhibit a GSD system with ZW-type female heterogamety (Ezaz et al. 2010). The $\mathrm{W}$ chromosome exhibits various stages of differ- entiation in this lineage. Whereas almost all lacertid lizards have large acrocentric $\mathrm{Z}$ and $\mathrm{W}$ chromosomes, the sand lizard (Lacerta agilis) has a large acrocentric $\mathrm{Z}$ chromosome and a micro-W chromosome (Odierna et al. 1993; Olmo and Signorino 2005).

In this study, we first performed chromosome banding and fluorescent in situ hybridization (FISH) with the $18 \mathrm{~S}-28 \mathrm{~S}$ and $5 \mathrm{~S}$ ribosomal RNA (rRNA) genes to identify the sex chromo- somes of L. agilis precisely. We also used FISH mapping of functional genes to construct a comparative cytogenetic map of $L$. agilis and examined the homology of the L. agilis chromosomes with the chromosomes of other Toxicofera species (E. quadrivirgata, V. salvator macromaculatus, $L$. reevesii rubritaeniata, and $A$. carolinensis) as well as the chicken. Our comparative mapping data enabled us to both identify the Z-linked genes of L. agilis and delineate the process of karyotype evolution in the lineage of Lacertidae.

\section{Materials and methods}

Specimens, cell culture, and chromosome preparation

The one male and one female L. agilis specimens analyzed were each captured in Asketunnan, which is situated ca. $50 \mathrm{~km}$ south of Gothenburg on the west coast of Sweden. After intraperitoneal injection of pentobarbital, the heart, lungs, and mesentery of each animal were removed and used for cell culture. The sex of each animal was identified mor- phologically and confirmed by its internal anatomy. All ex- perimental procedures using animals conformed to guidelines established by the Animal Care and Use Committee, Nagoya University, Japan. The tissues were minced and cultured in Dulbecco's modified Eagle's medium (Life Technologies- Gibco, Carlsbad, CA, USA) that was supplemented with $15 \%$ fetal bovine serum (Life Technologies-Gibco), $100 \mu \mathrm{g} / \mathrm{mL}$ kanamycin, and $1 \%$ antibiotic-antimycotic (Life Technologies-Gibco). The cultures were incubated at $26{ }^{\circ} \mathrm{C}$ in a humidified atmosphere of $5 \% \mathrm{CO}_{2}$ in air. Primary cultured fibroblasts were harvested using trypsin and then subcultured. For chromosome preparation, fibroblasts at the logarithmic phase of the growth cycle were incubated with 5- bromo-2'-deoxyuridine $(12 \mu \mathrm{g} / \mathrm{mL}$ ) (Sigma-Aldrich, St. Louis, MO, USA) for $12 \mathrm{~h}$, including 45 min of treatment with colcemid ( $120 \mathrm{ng} / \mathrm{mL}$ ) (Nacalai Tesque, Kyoto, Japan) before harvesting. Chromosome preparations were made using a standard air-drying method. For replication banding, the slides were stained with Hoechst 33258 ( 1 $\mu \mathrm{g} / \mathrm{mL}$ ) for $8 \mathrm{~min}$, heated at $65^{\circ} \mathrm{C}$ for $3 \mathrm{~min}$, and then exposed to ultraviolet light at $65^{\circ} \mathrm{C}$ for an additional 6 min (Matsuda and Chapman 1995). The slides were kept at $-80^{\circ} \mathrm{C}$ until use.

Molecular cloning of cDNA functional gene fragments The cDNA fragments of 30 functional genes were cloned from a male L. agilis using the PCR primers shown in Supplementary Table 1. Testes were homogenized and lysed with TRIzol 
Reagent (Life Technologies, Carlsbad, CA, USA), and total RNA was extracted following the manufac- turer's instructions. The cDNA fragments were obtained by RT-PCR using Oligo(dT) ${ }_{12-18}$ Primer and SuperScript II RNase $\mathrm{H}^{-}$Reverse Transcriptase (Life Technologies) and used as PCR templates to amplify L. agilis homologs of L. reevesii rubritaeniata and E. quadrivirgata genes that were mapped in our previous studies (Matsubara et al. 2006, 2012; Srikulnath et al.2009b). The cDNA amplification was carried out in $20 \mu \mathrm{l}$ of $1 \times$ ExTaq buffer that contained $1.5 \mathrm{mM} \mathrm{MgCl}_{2}$, $0.2 \mathrm{mM}$ dNTPs, $5.0 \mu \mathrm{M}$ degenerate primers, and $0.25 \mathrm{U}$ of TaKaRa Ex Taq (Takara Bio, Otsu, Japan). The temperature conditions used for PCR involved an initial denaturation step at $94{ }^{\circ} \mathrm{C}$ for $2 \mathrm{~min}$; followed by 35 cycles that each involved incubation at $94{ }^{\circ} \mathrm{C}$ for $30 \mathrm{~s}, 52^{\circ} \mathrm{C}$ for $30 \mathrm{~s}$, and $72{ }^{\circ} \mathrm{C}$ for $35 \mathrm{~s}$; and a final extension step at $72{ }^{\circ} \mathrm{C}$ for $10 \mathrm{~min}$. The PCR products were cloned using pGEM-T Easy Vector System I (Promega, Madison, WI, USA). Nucleotide sequences of the cDNA fragments were determined using an ABI 3130 Automated Capillary DNA Sequencer (Life TechnologiesApplied Biosystems, Carlsbad, CA, USA). The nucleotide sequences were used to search for homologies with those of the chicken in the National Center for Biotechnology Information (NCBI) database using the BLASTx and BLASTn programs (http://blast.ncbi.nlm.nih.gov/Blast. cgi) and were deposited in the DNA Data Bank of Japan (http://www.ddbj.nig.ac.jp/index-e.html) (Table 1). All cDNA fragments were confirmed to be homologs of chicken genes.

\section{FISH mapping}

The chromosomal locations of 86 functional genes and the 18S-28S and 5S rRNA genes were determined by FISH, as previously described (Matsuda and Chapman 1995; Srikulnath et al. 2009a), using cDNA fragments cloned from L. agilis (30 genes), L. reevesii rubritaeniata (54 genes; Srikulnath et al. 2009a, b), G. hokouensis (one gene; Srikulnath et al., unpublished data), and E. quadrivirgata (one gene; Matsubara et al., unpublished data) (Table 1), a partial 1.8-kb genomic DNA fragment (pCSI1) of the 8.2-kb fragment (EU727190) of C. siamensis 18S-28S rRNA genes, and a 99-base-pair (bp) genomic DNA fragment (pCSI5S; EU723235) of C. siamensis 5S rRNA genes.

We labeled $250 \mathrm{ng}$ of the 18S-28S rDNA probe with biotin-16-dUTP (Roche Diagnostics, Basel, Switzerland) by nick translation following the manufacturer's protocol (Roche Diagnostics). After hybridization of biotin-labeled 18S-28S rRNA to both male and female $L$. agilis chromosomes, the probes were stained with avidin labeled with fluorescein isothiocyanate (avidin-FITC; Vector Laboratories, Burlingame, CA, USA). Slides were subsequently counterstained with $0.75 \mu \mathrm{g} / \mathrm{mL}$ propidium iodide (PI). For chromosomal locali- zation of functional genes and the $5 \mathrm{~S}$ rRNA genes, we hybridized biotin-labeled cDNA fragments and 5S rDNA probe to L. agilis chromosomes. After hybridization, the probes were reacted with goat anti-biotin antibody (Vector Laboratories) and stained with Alexa Fluor ${ }^{8} 488$ rabbit anti- goat IgG (H+ L) conjugate (Life Technologies-Molecular Probes).

\section{Results}

The karyotype of Lacerta agilis

We examined more than 10 Hoechst-stained metaphase spreads of one male and one female L. agilis. Chromosome numbers were $2 n=38$ in both the male and the female. The male karyotype comprised 10 pairs of large acrocentric chromosomes (1st-10th), eight pairs of small acrocentric chromosomes (11th-18th), and one pair of microchromosomes (19th) (Fig. 1a). By contrast, the number of large acrocentric chromosomes was 19, and one additional microchromosome was found in the female (Fig. 1b). The Hoechst-stained chromosome banding pattern indicated that the fifth largest chromo- some was the $\mathrm{Z}$ sex chromosome and that the additional microchromosome was the $\mathrm{W}$ sex chromosome. Cytogenetic map of L. agilis and chromosome homology between L. agilis and chicken

The 18S-28S rRNA genes were localized to the subtelomeric region of chromosome 6 of L. agilis (Fig. 2a, c), and hybridization signals of the 5S rRNA genes were found in the terminal region of chromosome 7 of L. agilis (Fig. 2d-g). Analysis of 86 functional genes by FISH mapping with cDNA clones enabled us to construct a cytogenetic map of L. agilis (Figs. 3, 4, and 5). This is the first cytogenetic map for lacertid lizards. More than 30 metaphase spreads were observed for each gene, with hybridization efficiencies that ranged from approximately 20 to $80 \%$. Chromosome homology between

L. agilis and chicken was examined using the chicken genome database (http://www.ncbi.nlm.nih.gov/genome/guide/ chicken/). Seven genes that were mapped to L. agilis (LAG) chromosome 1 were localized to chicken (Gallus gallus, GGA) chromosomes 5, 7, and 19 (Figs. 3 and 5, Table 1). Seven genes mapped on LAG2 were localized to GGA12, 13, 16, and 18. Three genes on LAG3 were located on GGA3 and 13. LAG4 corresponded to GGA1q, and LAG5 (the Z sex chromosome) had homology with GGA6 and GGA9 (Figs. 4, 5). Six genes on LAG6 were localized to GGA17, 21, and 23, and six genes on 
LAG7 were localized to GGA8, 20, and 26. LAG8 was homologous to GGA2q; however, HDAC3 on LAG8 was located on GGA13. LAG9 showed homology with GGA1p and LAG10 with GGA4q. The homologous segments of 16 chicken genes linked to microchromosomes were found in small acrocentric chromosomes (LAG11-18) and a microchromosome (LAG19) (Table 1).

\section{Discussion}

Homology of the L. agilis $\mathrm{Z}$ chromosome with chromosomes of other Toxicofera species

The karyotype of female L. agilis $(2 \mathrm{n}=38)$ was found to consist of nine pairs of large acrocentric chromosomes, eight pairs of small acrocentric chromosomes, one pair of microchromosomes, a large acrocentric $\mathrm{Z}$ chromosome, and a micro-W chromosome. This result is identical to those of previous reports (De Smet 1981; Odierna et al. 1993). The L. agilis $\mathrm{Z}$ sex chromosome was identified as the fifth largest acrocentric chromosome. Comparative gene mapping also revealed that the L. agilis Z chromosome has homology with chicken chromosomes 6 and 9 (GGA6 and GGA9) (Fig. 5). The fusion of GGA6 and GGA9 is also found in

A. carolinensis chromosome 3 (Alföldi et al. 2011). In addi- tion, the L. agilis Z chromosome was shown to be homologous to a single pair of autosomes in three reptilian species: chromosome 3p in L. reevesii rubritaeniata (LRE3p), chromosome 5 in E. quadrivirgata (EQU5), and chromosome 6 in

V. salvator macromaculatus (VSA6) (Figs. 5, 6), which indi- cates that this fusion occurred at least in the common ancestor of Toxicofera and Lacertoidea (Episquamata) (Pyron et al. 2013). The L. agilis Z chromosome showed no homology with either the Z chromosome of G. hokouensis (Gekkota), which is homologous to GGAZ (Kawai et al. 2009), or the $\mathrm{X}$ chromosome of A. carolinensis (Iguania), which is homologous to GGA15 (Alföldi et al. 2011). Moreover, the Z chromosome of Pogona vitticeps (Iguania) has homology with GGA23, and the $\mathrm{Z}$ chromosome of snakes is homologous to GGA2 and

27 (Matsubara et al. 2006, 2012; Ezaz et al. 2013). These results suggest that the sex chromosomes of these squamate reptiles differentiated independently in each lineage within the same order from different autosomal pairs of the common ancestor.

In Lacertidae, some species have homomorphic sex chro- mosomes, whereas many other lacertid lizards show morphologically differentiatedZW-type sex chromosomes (Olmo and Signorino 2005). For instance, large-sized W sex chromosomes are found in Takydromus sexlineatus and Gallotia galloti, whereas Acanthodactylus erythrurus, Meroles cuneirostris, Lacerta monticola, and Lacerta viridis have intermediate-sized W chromosomes, and Lacerta dugesii and Eremias velox have micro-W chromosomes (Olmo et al. 1987; Pokorná et al. 2011). These findings indicate that the extent of $\mathrm{W}$ chromosome differentiation varies even within the same family. L. agilis was also shown to have a micro-W chromosome in which telomeric TTAGGG repeats were am- plified after extensive differentiation of the W chromosome (Matsubara et al., unpublished data). This suggests that, of the sex chromosomes that have been characterized in lacertid lizards, the micro-W chromosome of $L$. agilis shows the highest level of differentiation. This mixture of homomorphic and heteromorphic sex chromosomes in lacertid lizards is common in vertebrates; for example, similar patterns are also observed in Anolis and Sceloporus lizards, in which morphologically indistinguishable homomorphic sex chromo- somes and male heterogametic XY and XXY sex chromo- somes coexist within the same genus (Leaché and Sites 2009; Gamble et al. 2013).

The process of karyotype evolution in the lineage of Lacertidae

The cytogenetic map comparing $L$. agilis with the chicken and three squamate reptiles revealed that nine chicken macrochromosomes and/or macrochromosome segments (GGA1p, 1q, 2q, 3, 4q, 5, 6, 7, and 8) are highly conserved in eight macrochromosomes of $L$. agilis [LAG1, 3, 4, 5(Z), 7, 8, 9, and 10] as well as E. quadrivirgata (EQU), V. salvator macromaculatus (VSA), and L. reevesii rubritaeniata (LRE) macrochromosomes (Matsubara et al. 2006, 2012; Srikulnath et al. 2009b, 2013; Uno et al. 2012) (Figs. 5, 6). These conserved syntenies with chicken chromosomes were also found in A. carolinensis (ACA) macrochromosomes (Alföldi et al. 2011), although half of the gene markers mapped in this study have not been localized in the A. carolinensis genome. However, the genes mapped on LRE2p, LRE6, EQU2p, EQUZ, VSA1p, VSA4, ACA2, and ACA6 were all localized to small acrocentric chromosomes of L. agilis (LAG11-18), which indicates that rearrangement patterns of these segments differ between Toxicofera and Lacertoidea(Table 1). The homologous segments of 39 chicken microchromosome-linked genes were found in LAG1, 2, 3, Z(5), 6, 7, and 8, small acrocentric chromosomes (LAG11- 
18), and a microchromosome (LAG19) (Fig. 5, Table 1). The fusion of GGA17, GGA21, and GGA23 was found in LAG6; by contrast, these three homologous segments were located on microchromosomes of E. quadrivirgata and

$V$. salvator macromaculatus, which suggests that the fusion of these three chicken microchromosomes might have only oc- curred in L. agilis (Table 1).

The 18S-28S rRNA genes were localized to the subtelomeric region of chromosome 6 in L. agilis. Chromosomal locations of nucleolar organizer region (NOR) and 18S-28S rRNA genes have been reported in several lacertid species and are categorized into three types: terminal region on the long arm of a large acrocentric chromosome pair, terminal region on the long arm of a small acrocentric chromosome pair, and microchromosome (Odierna et al. 1987, 1995; Olmo et al. 1991; Olmo and Signorino 2005). In Aves, the major rRNA genes are supposed to have been located on a single pair of microchromosomes in the ancestral avian karyotype (Nishida-Umehara et al. 2007). The chromosomal location of the major rRNA genes in the ancestral karyotype of Toxicofera and Lacertoidea has still not been de- fined; however, the present results strongly support the assertion that the disappearance of microchromosomes in lacertid lizards resulted from repeated fusions of microchromosomes that existed in the ancestral karyotype. The microchromosome-linked genes of V. salvator macromaculatus and L. reevesii rubritaeniata, TRIM37 and $A M H$ (GGA19-linked gene homologs) and EEF2 (GGA28-linked gene homologs), were shown to be localized to the distal ends of the short and long arms of EQU1, respectively (Matsubara et al. 2006, 2012; Srikulnath et al. 2009b, 2013). However, in L. agilis, TRIM37 and AMH were shown to be localized to the interstitial region of LAG1 that is homologous to EQU $1 \mathrm{q}$, and EEF 2 was located on one pair of microchromosomes (LAG19) (Fig. 6). These results suggest that this tandem fusion of microchromosomes with macrochromosomes occurred in E. quadrivirgata independently from the lineage of $L$. agilis.

Cytogenetic maps that compare L. agilis with each of three Toxicofera species enabled us to apply the most parsimonious explanation of chromosomal rearrangements to deduce the course of karyotype evolution in lacertid species (Fig. 6). LAG3 was shown to be homologous to LRE1p, EQU1p, and VSA2p; however, the gene order from the centromere differed between $L$. agilis and these three species. This result suggests that two paracentric inversions occurred in the proto-LAG3 after centric fission from the proto bi-armed chromosome, which seems to be the ancestral type of LRE1, EQU1, and VSA2 (Fig. 7a). Alternative possibilities are that two inversions oc- curred sequentially in LAG3, and this chromosome then fused with LAG1 (Fig. 7a), or that two inversions occurred sequentially in the $\mathrm{p}$ arm of the proto bi-armed chromosome after centric fusion of LAG3 and LAG1 or before centric fission of the proto bi-armed chromosome (Fig. 7b). According to the most parsi- monious explanation of chromosomal rearrangements in the lineages of Toxicofera and Lacertoidea, the fission event to form LAG1 and LAG3 from the proto bi-armed chromosome is most likely. LAG4 was shown to correspond to LRE3q and LAGZ (5) to LRE3p. LAG7 was homologous to LRE4q and EQU3q and LAG8 to LRE4p and EQU3p. LAG9 was homologous to LRE5q and LAG10 to LRE5p. These six acrocentric chromo- somes in L. agilis might each have been derived from centric fission of an ancestral bi-armed chromosome, or alternatively, the bi-armed chromosomes may each have resulted from centric fusion of two acrocentric chromosomes (Fig. 7c-e). However, centric fusion between LAG4 and LAGZ (5) and centric fission of LRE4 or EQU3 and of LRE5 are most likely because LAG4 and LAGZ (5), LRE4 or EQU3, and LRE5 are considered to be the prototypes in the lineages of Toxicofera and Lacertoidea according to the most parsimonious explanation of chromosomal rearrangements.

Comparison of the cytogenetic maps of four squamate spe- cies (L. agilis, E. quadrivirgata, V. salvator macromaculatus, L. reevesii rubritaeniata, and $A$. carolinensis) in this study revealed the origin of the $\mathrm{Z}$ and $\mathrm{W}$ sex chromosomes of L. agilis and the karyotype reorganization that occurred be- tween L. agilis and other Toxicofera species. However, comparative gene mapping for more closely related species in Lacertoidea, such as Gymnophthalmidae and Amphisbaenia, and also distantly related species in Gekkota, which lack microchromosomes, is necessary in order to delineate karyo- type evolution in Squamata more precisely. Greater precision in the identification of conserved chromosomal segments in sauropsids will provide insight into the phylogenetic hierarchy of genome evolution in amniotes. 
Table 1 List of $86 \mathrm{cDNA}$ clones mapped to sand lizard (Lacerta agilis) chromosomes and their chromosomal locations in the water monitor lizard (Varanus salvator macromaculatus), the butterfly lizard (Leiolepis reevesii rubritaeniata), the Japanese four-striped rat snake (Elaphe quadrivirgata), the green anole (Anolis carolinensis), and chicken (Gallus gallus)

\begin{tabular}{|c|c|c|c|c|c|c|c|c|}
\hline \multirow{2}{*}{$\begin{array}{l}\text { Gene } \\
\text { symbol }\end{array}$} & \multirow{2}{*}{$\begin{array}{l}\text { Sequenced length } \\
\text { of cDNA } \\
\text { fragment (bp) }\end{array}$} & \multicolumn{6}{|c|}{ Chromosomal location } & \multirow{2}{*}{$\begin{array}{l}\text { Accession } \\
\text { number }\end{array}$} \\
\hline & & L. agilis & V. salvator macromaculatus & $\begin{array}{l}\text { L. reevesii } \\
\text { rubritaeniata }\end{array}$ & E. quadrivirgata & A. carolinensis & G. gallus & \\
\hline$A C V R 1^{\mathrm{a}}$ & 845 & 1 & $2 q$ & - & - & - & 7 & AB794073 \\
\hline$C A C N B 4^{\mathrm{b}}$ & 882 & 1 & $2 q$ & $1 q 12.2-q 13.1$ & $1 \mathrm{q}$ & - & 7 & AB490346 \\
\hline TRIM37 $7^{\mathrm{b}, \mathrm{c}}$ & 631,577 & 1 & Micro & Micro & - & - & 19 & AB490382, AB490383 \\
\hline$A M H^{\mathrm{d}, \mathrm{c}}$ & 712,709 & 1 & Micro & - & - & - & 19 & AB794387, AB794388 \\
\hline$W T 1^{\mathrm{b}}$ & 542 & 1 & $2 q$ & $1 \mathrm{q} 21.1-\mathrm{q} 22.1$ & $1 q$ & 1 & 5 & AB490347 \\
\hline$D Y N C 1 H 1^{\mathrm{b}}$ & 997 & 1 & $2 q$ & $1 \mathrm{q} 32.1-\mathrm{q} 32.3$ & $1 q$ & - & 5 & AB490348 \\
\hline CYP2C21-like & 1,331 & 1 & $2 q$ & - & - & - & - & AB794068 \\
\hline$K R T 8^{\mathrm{a}}$ & 835 & 2 & Micro & - & - & - & - & AB794081 \\
\hline$S O X 9^{\mathrm{b}, \mathrm{c}}$ & 603,717 & 2 & $1 \mathrm{q}$ & $2 \mathrm{q} 11.2-\mathrm{q} 11.4$ & $2 q$ & - & 18 & AB490350, AB490351 \\
\hline$T O B 1^{\mathrm{a}}$ & 950 & 2 & $1 \mathrm{q}$ & - & - & 2 & 18 & AB794087 \\
\hline$R U F Y 1^{\mathrm{b}}$ & 545 & 2 & $1 q$ & $2 \mathrm{q} 12.2-\mathrm{q} 21.1$ & $2 q$ & 2 & 13 & AB490352 \\
\hline$T K T^{\mathrm{b}}$ & 943 & 2 & $1 q$ & $2 \mathrm{q} 11.1$ & $2 q$ & 2 & 12 & AB490349 \\
\hline$A L A S 1^{\mathrm{a}}$ & 1,060 & 2 & $1 q$ & - & - & 2 & 12 & AB794074 \\
\hline$B R D 2^{\mathrm{b}}$ & 691 & 2 & $1 q$ & $2 \mathrm{q} 22.2$ & - & - & 16 & AB480353 \\
\hline$X A B 1^{\mathrm{b}}$ & 489 & 3 & $2 p$ & $1 \mathrm{p} 22.4$ & $1 \mathrm{p}$ & - & 3 & AB490344 \\
\hline$F B X W 11^{\mathrm{e}}$ & 926 & 3 & $2 p$ & - & $1 \mathrm{p}$ & 1 & 13 & AB792691 \\
\hline$E S R I^{\mathrm{b}}$ & 951 & 3 & $2 p$ & $1 \mathrm{p} 21.2$ & $1 \mathrm{p}$ & - & 3 & AB490345 \\
\hline$T P T 1^{\mathrm{b}}$ & 438 & 4 & $5 q$ & $3 p 11.1-q 11$ & $4 p$ & - & $1 \mathrm{q}$ & AB490359 \\
\hline$I P O 5^{\mathrm{a}, \mathrm{c}}$ & 703,492 & 4 & $5 q$ & - & - & 3 & $1 \mathrm{q}$ & AB793729, AB793730 \\
\hline$E I F 2 S 3^{\mathrm{b}}$ & 733 & 4 & $5 \mathrm{q}$ & $3 q 12.3-q 21.1$ & $4 p$ & 3 & $1 \mathrm{q}$ & AB490361 \\
\hline$O C A 2^{\mathrm{b}}$ & 782 & 4 & $5 \mathrm{q}$ & $3 q 12.1-q 12.2$ & - & 3 & $1 \mathrm{q}$ & AB490360 \\
\hline$E L M O D 1^{\mathrm{b}}$ & 682 & 4 & $5 \mathrm{q}$ & $3 q 22.1-q 22.3$ & $4 q$ & - & $1 \mathrm{q}$ & AB490362 \\
\hline$A D A M 12^{\mathrm{a}}$ & 933 & $5(\mathrm{Z})$ & $6 q$ & - & - & 3 & 6 & AB794067 \\
\hline$P S A P^{\mathrm{b}}$ & 1,325 & $5(\mathrm{Z})$ & $6 \mathrm{q}$ & $3 \mathrm{p} 11.2-\mathrm{p} 12.2$ & $5 q$ & - & 6 & AB490358 \\
\hline$B T R C^{\mathrm{b}}$ & 889 & $5(\mathrm{Z})$ & $6 \mathrm{q}$ & $3 \mathrm{p} 21.1-\mathrm{p} 21.2$ & - & - & 6 & AB490357 \\
\hline$S H 3 P X D 2 A^{\mathrm{b}}$ & 1,210 & $5(\mathrm{Z})$ & $6 \mathrm{q}$ & $3 \mathrm{p} 22.1$ & $5 \mathrm{q}$ & 3 & 6 & AB490356 \\
\hline$S L I T 1^{\mathrm{a}}$ & 995 & $5(\mathrm{Z})$ & $6 q$ & - & - & - & 6 & AB794071 \\
\hline$E P H A 4^{\mathrm{a}}$ & 888 & $5(\mathrm{Z})$ & $6 q$ & - & - & 3 & 9 & AB794079 \\
\hline$T L O C 1^{\mathrm{b}}$ & 782 & $5(\mathrm{Z})$ & $6 \mathrm{q}$ & $3 \mathrm{p} 22.3$ & $5 q$ & 3 & 9 & AB490355 \\
\hline$S K I L^{\mathrm{a}}$ & 1,686 & $5(\mathrm{Z})$ & $6 q$ & - & - & 3 & 9 & AB794070 \\
\hline$N C L^{\mathrm{a}}$ & 1,780 & $5(\mathrm{Z})$ & $6 q$ & - & - & - & 9 & AB794069 \\
\hline
\end{tabular}


Table 1 (continued)

\begin{tabular}{|c|c|c|c|c|c|c|c|c|}
\hline \multirow{2}{*}{$\begin{array}{l}\text { Gene } \\
\text { symbol }\end{array}$} & \multirow{2}{*}{$\begin{array}{l}\text { Sequenced length } \\
\text { of cDNA } \\
\text { fragment (bp) }\end{array}$} & \multicolumn{6}{|c|}{ Chromosomal location } & \multirow{2}{*}{$\begin{array}{l}\text { Accession } \\
\text { number }\end{array}$} \\
\hline & & L. agilis & V. salvator macromaculatus & $\begin{array}{l}\text { L. reevesii } \\
\text { rubritaeniata }\end{array}$ & E. quadrivirgata & A. carolinensis & G. gallus & \\
\hline$E N O 1^{\text {a }}$ & 917 & 6 & Micro & - & Micro & - & 21 & AB794078 \\
\hline$T M E M 57^{\mathrm{a}}$ & 800 & 6 & Micro & - & - & - & 23 & AB794086 \\
\hline$R N F 19 B^{\mathrm{a}}$ & 942 & 6 & Micro & - & - & - & 23 & AB793732 \\
\hline$D N M 1^{\mathrm{a}}$ & 1,014 & 6 & Micro & - & Micro & - & 17 & AB794076 \\
\hline$P P P 2 R 1 A^{\mathrm{a}}$ & 1,169 & 6 & Micro & - & - & - & - & AB793731 \\
\hline$G R I N 1^{\mathrm{a}}$ & 893 & 6 & Micro & - & Micro & - & 17 & AB794080 \\
\hline$R P E 65^{\mathrm{a}}$ & 1,130 & 7 & $8 \mathrm{p}$ & - & - & - & 8 & AB793733 \\
\hline ZNF326 & 892 & 7 & $8 p$ & $4 q 12.1-q 12.3$ & $3 q$ & 4 & 8 & AB490366 \\
\hline$C N T N 2^{\mathrm{a}}$ & 922 & 7 & $8 \mathrm{q}$ & - & - & 4 & 26 & AB793728 \\
\hline$U S P 49^{\mathrm{a}}$ & 1,210 & 7 & $8 \mathrm{q}$ & - & - & 4 & 26 & AB794088 \\
\hline$R B M 12^{\mathrm{b}}$ & 943 & 7 & $8 \mathrm{q}$ & $4 q 21.2-q 22.1$ & $3 q$ & 4 & 20 & AB490367 \\
\hline$R P N 2^{\mathrm{a}}$ & 1,229 & 7 & $8 \mathrm{q}$ & - & - & - & 20 & AB794084 \\
\hline$H D A C 3^{\mathrm{b}}$ & 929 & 8 & $7 \mathrm{p}$ & 4p11.1-p11.2 & $3 p$ & 4 & 13 & AB490365 \\
\hline$S S 18^{\mathrm{b}, \mathrm{c}}$ & 614,508 & 8 & $7 \mathrm{q}$ & $4 p 12.2-p 21.2$ & $3 p$ & 4 & $2 q$ & AB490364, AB490397 \\
\hline$E N P P 2^{\mathrm{b}}$ & 961 & 8 & $7 \mathrm{q}$ & $4 \mathrm{p} 22.2-\mathrm{p} 22.4$ & $3 p$ & - & $2 q$ & AB490363 \\
\hline$D Y R K 2^{\mathrm{b}}$ & 1,086 & 9 & $3 p$ & $5 q 12-q 21.2$ & - & - & $1 \mathrm{p}$ & AB490373 \\
\hline$R A N G A P 1^{\mathrm{b}}$ & 1,024 & 9 & $3 p$ & $5 \mathrm{q} 21.2-\mathrm{q} 22.1$ & $6 \mathrm{q}$ & 5 & $1 \mathrm{p}$ & AB490374 \\
\hline$T T C 26^{\mathrm{b}}$ & 772 & 9 & $3 p$ & $5 q 21.3-q 22.3$ & $6 \mathrm{q}$ & - & $1 \mathrm{p}$ & AB490375 \\
\hline$S O X 5^{\mathrm{b}, \mathrm{c}}$ & 851,705 & 9 & $3 p$ & $5 q 22.1-q 22.4$ & - & 5 & $1 \mathrm{p}$ & AB490376, AB490377 \\
\hline$U C H L 1^{\mathrm{b}}$ & 595 & 10 & $3 p$ & 5p11.1-p12.1 & $6 \mathrm{p}$ & 5 & $4 \mathrm{q}$ & AB490372 \\
\hline$E X O C 1^{\mathrm{b}}$ & 1,177 & 10 & $3 q$ & $5 \mathrm{p} 11.2-\mathrm{p} 12.2$ & $7 p$ & 5 & $4 \mathrm{q}$ & AB490371 \\
\hline$A C S L 1^{\mathrm{b}}$ & 748 & 10 & $3 q$ & $5 \mathrm{p} 12.1-\mathrm{p} 12.2$ & $7 q$ & 5 & $4 q$ & AB490370 \\
\hline$D C L K 2^{\mathrm{b}}$ & 688 & 10 & $3 q$ & $5 \mathrm{p} 12.1-\mathrm{p} 21$ & - & 5 & $4 \mathrm{q}$ & AB490369 \\
\hline$S M A D 1^{\mathrm{a}}$ & 944 & 10 & $3 q$ & - & - & 5 & $4 q$ & AB794085 \\
\hline$R A P 1 G D S 1^{\mathrm{b}}$ & 991 & 10 & $3 q$ & $5 \mathrm{p} 21-\mathrm{p} 22.2$ & $7 \mathrm{q}$ & - & $4 q$ & AB490368 \\
\hline$A T P 5 A 1^{\mathrm{f}}$ & 987 & $11-18$ & $1 \mathrm{p}$ & $2 \mathrm{p} 23.1-\mathrm{p} 23.3$ & $2 p$ & 1 & $\mathrm{Zp}$ & AB480291, AB480292 \\
\hline$G H R^{\mathrm{b}}$ & 771 & $11-18$ & $1 \mathrm{p}$ & $2 \mathrm{p} 22-\mathrm{p} 23.3$ & $2 p$ & 2 & $\mathrm{Zp}$ & AB480290 \\
\hline$C H D 1^{\mathrm{b}}$ & 961 & $11-18$ & $1 \mathrm{p}$ & $2 \mathrm{p} 21$ & $2 p$ & 2 & $\mathrm{Zq}$ & AB480289 \\
\hline$D M R T 1^{\mathrm{b}}$ & 628 & $11-18$ & $1 \mathrm{p}$ & $2 \mathrm{p} 12-\mathrm{p} 21$ & $2 p$ & 2 & $\mathrm{Zp}$ & AB480288 \\
\hline$R_{P S \sigma^{\mathrm{b}}}$ & 521 & $11-18$ & $1 \mathrm{p}$ & $2 \mathrm{p} 11.3-\mathrm{p} 12$ & $2 p$ & 2 & $\mathrm{Zp}$ & $\mathrm{AB} 480287$ \\
\hline$A C O 1 / I R E B P^{\mathrm{f}}$ & 1,122 & $11-18$ & $1 \mathrm{p}$ & 2p11.2-p11.4 & $2 p$ & - & $\mathrm{Zq}$ & $\mathrm{AB} 480285, \mathrm{AB} 480286$ \\
\hline$M Y S T 2^{\mathrm{b}}$ & 1,261 & $11-18$ & $4 p$ & 6p21.1-p22.2 & $\mathrm{Zq}$ & 6 & 27 & AB490378 \\
\hline
\end{tabular}


Table 1 (continued)

\begin{tabular}{|c|c|c|c|c|c|c|c|c|}
\hline \multirow{2}{*}{$\begin{array}{l}\text { Gene } \\
\text { symbol }\end{array}$} & \multirow{2}{*}{$\begin{array}{l}\text { Sequenced length } \\
\text { of cDNA } \\
\text { fragment (bp) }\end{array}$} & \multicolumn{6}{|c|}{ Chromosomal location } & \multirow{2}{*}{$\begin{array}{l}\text { Accession } \\
\text { number }\end{array}$} \\
\hline & & L. agilis & V. salvator macromaculatus & $\begin{array}{l}\text { L. reevesii } \\
\text { rubritaeniata }\end{array}$ & E. quadrivirgata & A. carolinensis & G. gallus & \\
\hline $\operatorname{STAT3}^{\mathrm{a}}$ & 1,654 & $11-18$ & $4 p$ & - & - & 6 & 27 & AB793734 \\
\hline$T O P 2 A^{\mathrm{a}, \mathrm{c}}$ & 882,477 & $11-18$ & $4 p$ & - & - & 6 & 27 & AB793735, AB793736 \\
\hline$T O P 2 B^{\mathrm{a}}$ & 1,639 & $11-18$ & $4 \mathrm{q}$ & - & - & - & $2 p$ & AB793737 \\
\hline$C T N N B 1^{\mathrm{b}}$ & 1,201 & $11-18$ & $4 \mathrm{q}$ & $6 q 11$ & $\mathrm{Zp}$ & 6 & $2 p$ & AB490379 \\
\hline$W A C^{\mathrm{b}}$ & 1,122 & $11-18$ & $4 \mathrm{q}$ & $6 q 21-q 23$ & $\mathrm{Zp}$ & 6 & $2 p$ & AB490381 \\
\hline$G A D 2^{\mathrm{b}}$ & 672 & $11-18$ & $4 \mathrm{q}$ & $6 q 21-q 23$ & $\mathrm{Zp}$ & 6 & $2 p$ & AB490380 \\
\hline$C U L 4 B^{\mathrm{b}}$ & 723 & $11-18$ & Micro & Micro & - & - & $4 p$ & AB490387 \\
\hline$A T R X^{\mathrm{b}}$ & 804 & $11-18$ & Micro & Micro & Micro & - & $4 p$ & AB490386 \\
\hline$A R^{\mathrm{b}}$ & 941 & $11-18$ & Micro & Micro & Micro & - & $4 p$ & AB490385 \\
\hline$C H D 2^{\mathrm{b}, \mathrm{c}}$ & 654,692 & $11-18$ & Micro & Micro & - & - & 10 & AB490388, AB490389 \\
\hline$P O L G^{\mathrm{a}}$ & 1,565 & $11-18$ & Micro & - & Micro & - & 10 & AB794083 \\
\hline$B R D 7^{\mathrm{b}}$ & 784 & $11-18$ & Micro & Micro & Micro & - & 11 & AB490390 \\
\hline$E E F 2 K^{\mathrm{a}}$ & 970 & $11-18$ & Micro & - & Micro & - & 14 & AB794077 \\
\hline$U B N 1^{\mathrm{a}}$ & 1,153 & $11-18$ & Micro & - & - & - & 14 & AB794072 \\
\hline$P D X D C 1^{\mathrm{a}}$ & 1,619 & $11-18$ & Micro & - & - & - & 14 & AB794082 \\
\hline$N F 2^{\mathrm{b}}$ & 940 & $11-18$ & Micro & Micro & - & - & 15 & AB490393 \\
\hline$S F 3 A 1^{\mathrm{b}}$ & 937 & $11-18$ & Micro & Micro & - & - & 15 & AB490394 \\
\hline$A T P 2 A 2^{\mathrm{b}}$ & 972 & $11-18$ & Micro & Micro & Micro & Micro & 15 & AB490391 \\
\hline$S B N O 1^{\mathrm{b}}$ & 1,345 & $11-18$ & Micro & Micro & - & Micro & 15 & AB490392 \\
\hline$H S P A 8^{\mathrm{b}}$ & 906 & $11-18$ & Micro & Micro & Micro & Micro & 24 & AB490395 \\
\hline$A R N T^{\mathrm{a}}$ & 1,034 & $11-18$ & Micro & - & - & - & 25 & AB794075 \\
\hline$A C T N 4^{\mathrm{b}}$ & 1,069 & $11-18$ & Micro & Micro & - & - & - & AB490396 \\
\hline$E E F 2^{\mathrm{b}}$ & 975 & 19 & Micro & Micro & $1 \mathrm{q}$ & 1 & 28 & AB490384 \\
\hline
\end{tabular}

- No data

${ }^{\text {a }}$ The cDNA fragments were obtained from L. agilis in Srikulnath et al. (unpublished data)

${ }^{\mathrm{b}}$ The cDNA fragments were obtained from of L. reevesii rubritaeniata in Srikulnath et al. (2009a, b)

${ }^{\mathrm{c}}$ Nucleotide sequences of two accession numbers were determined separately by forward and reverse primers in one clone

${ }^{\mathrm{d}}$ The cDNA fragments were obtained from of E. quadrivirgata in Matsubara et al. (unpublished data)

${ }^{\mathrm{e}}$ The cDNA fragments were obtained from of G. hokouensis in Srikulnath et al. (unpublished data)

${ }^{\mathrm{f}}$ Nucleotide sequences of cDNA fragments of L. reevesii rubritaeniata were taken from Srikulnath et al. (2009b). For mapping of ATP5A1, and ACO1/IREBP, two different fragments of the gene were simultaneously hybridized to chromosomes 
Fig. 1 Hoechst-stained

karyotypes of L. agilis: a male,

(b) female. Scale bars represent

$10 \mu \mathrm{m}$

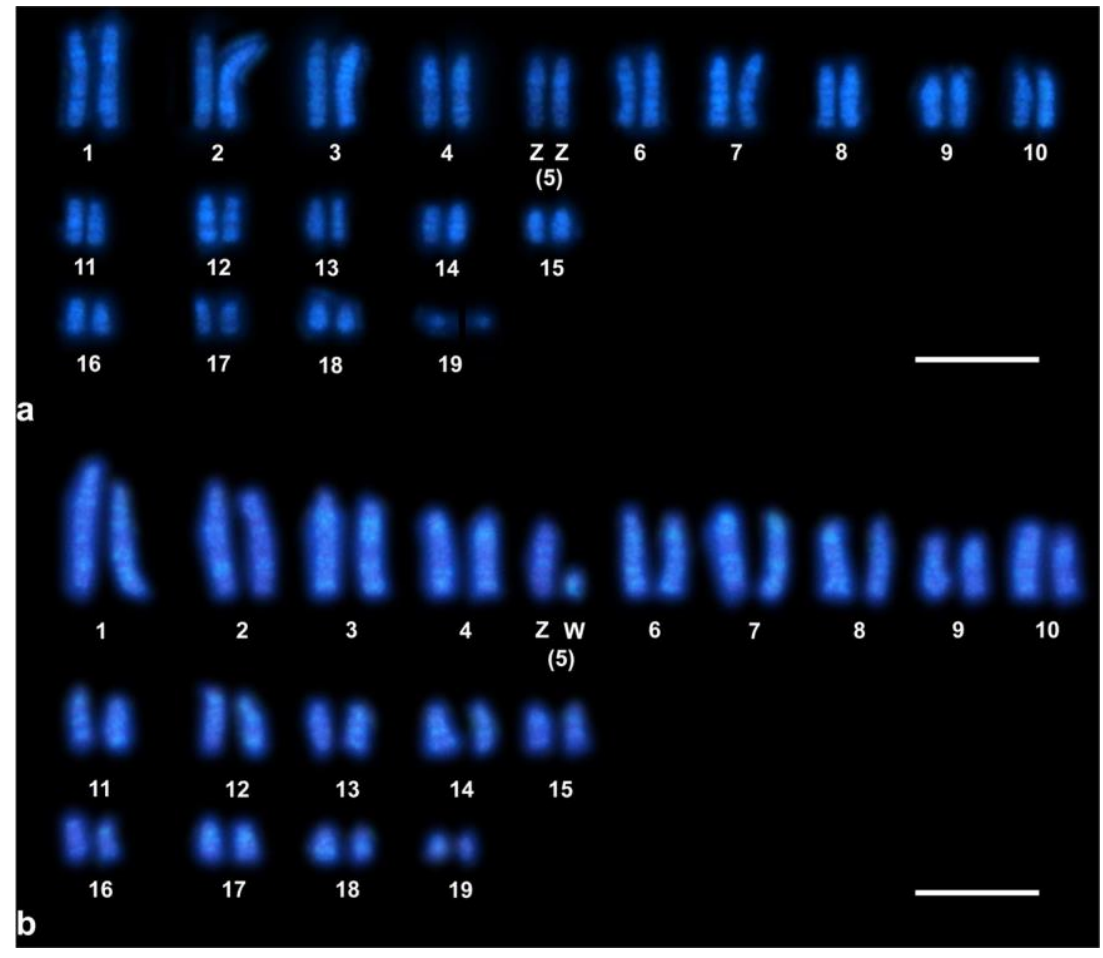




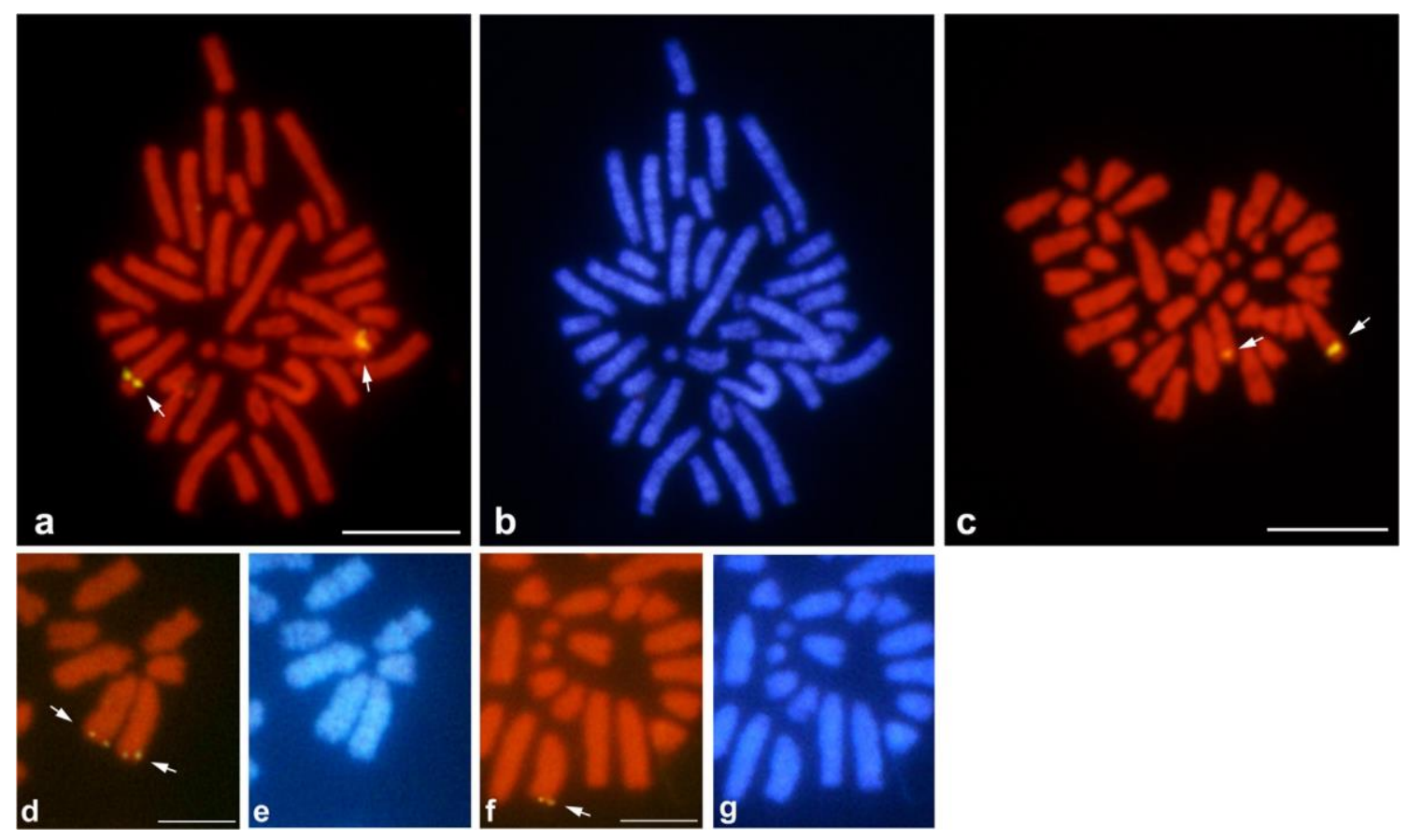

Fig. 2 Chromosomal locations of the 18S-28S and 5S rRNA genesin

L. agilis: a, b, d, e male, c, f, g female. a, c, d, f Hybridization patterns of the 18S-28S genes (a, c) and the 5S rRNA genes (d, f). b, e, g Hoechststained patterns of the same metaphase spreads are shown in (a, d, f), respectively. Arrows indicate the hybridization signals of the 18S-28S and 5S rRNA genes. Scale bars represent $10 \mu \mathrm{m}$ 


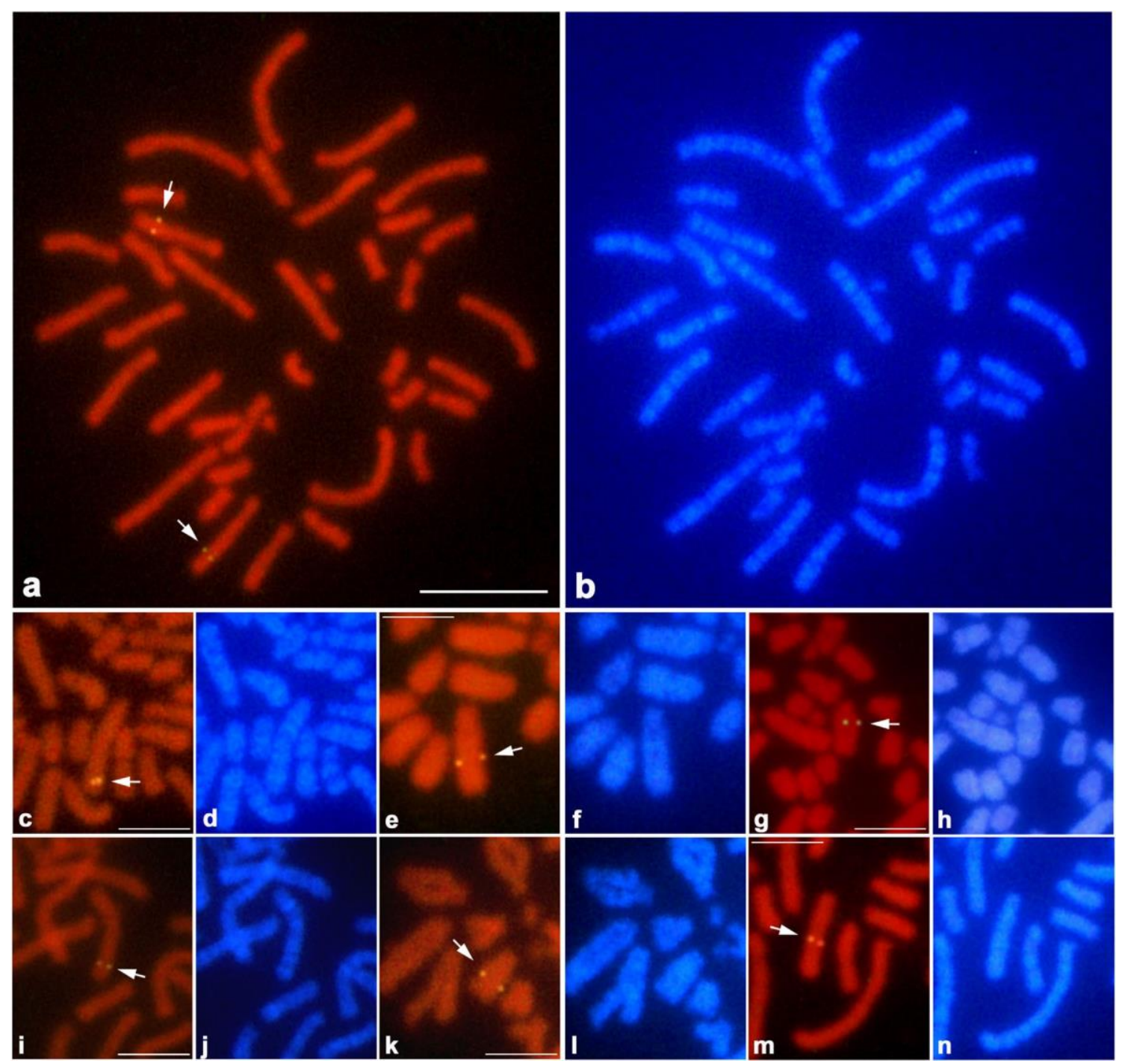

Fig. 3 Chromosomal locations of cDNA fragments of functional genes in male L. agilis. The DNM1 gene was localized to chromosome 6 (a), CYP2C21-like to chromosome 1 (c), ALAS1 to chromosome 2 (e), ZNF326 to chromosome 7 (g), ENPP2 to chromosome 8 (i), TTC26 to chromosome 9 (k), and $D C L K 2$ to chromosome $10(\mathrm{~m})$. b, d, f, h, j, l, n Hoechst-stained patterns of the same metaphase spreads are shown in (a, c, e, $\mathrm{g}, \mathrm{i}, \mathrm{k}, \mathrm{m})$, respectively. Arrows indicate the hybridization signals. Scale bars represent $10 \mu \mathrm{m}$ in (a) and $5 \mu \mathrm{m}$ in $(\mathrm{c}-\mathrm{n})$

Fig. 4 Chromosomal locations of cDNA fragments of chromosome 5 (Z)-linked genes in L. agilis. The PSAP (a), SH3PXD2A (c), EPHA4 (e), and TLOC1 (g) genes were localized to the $\mathrm{Z}$ sex chromosome. b, d, f, h Hoechststained patterns of the PI-stained metaphase spreads are shown in (a, c, e, g), respectively. Arrows indicate the hybridization signals. Scale bars represent $5 \mu \mathrm{m}$

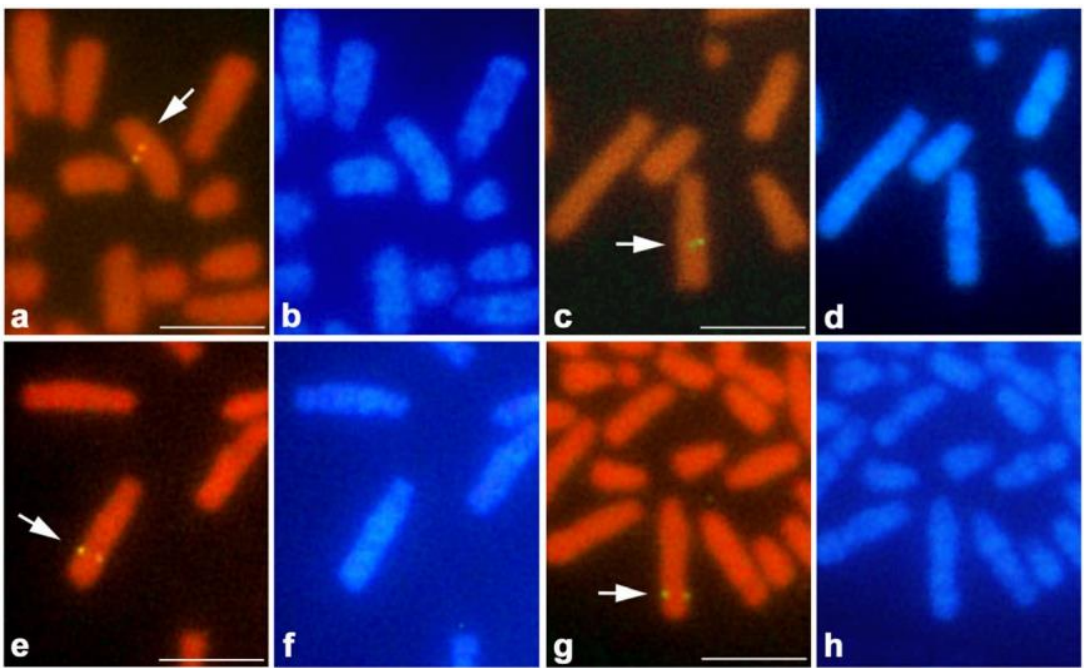




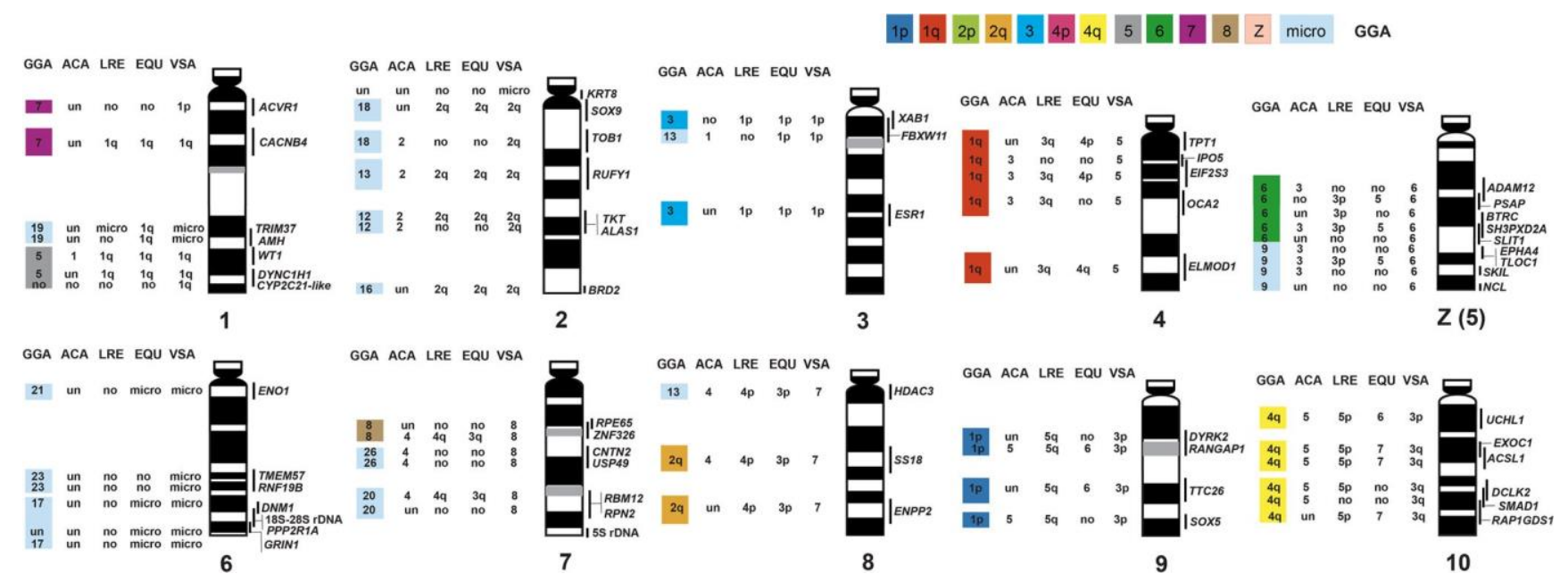

Fig. 5 Cytogenetic maps comparing L. agilis with the chicken, the green anole, butterfly lizard, Japanese four-striped rat snake, and water monitor lizard. All maps were constructed using 56 functional genes and the $18 \mathrm{~S}-28 \mathrm{~S}$ and $5 \mathrm{~S}$ rRNA genes. The ideogram of $L$. agilis chromosomes was constructed according to Hoechst-stained band patterns. Locations of the genes on L. agilis chromosomes are shown to the right of the chromosomes. The numbers of chromosome of chicken (Gallus gallus, GGA), the green anole (Anolis carolinensis, ACA), butterfly lizard (Leiolepis reevesii rubritaeniata, LRE), Japanese four-striped rat snake (Elaphe quadrivirgata, EQU), and water monitor lizard (Varanus salvator macromaculatus, VSA) that show homologies with L. agilis chromo- somes are shown to the left. Whereas "no" indicates no data on chromo- some homology, "un" indicates a gene whose chromosomal location remains undetermined. The chromosomal locations of genes for L. reevesii rubritaeniata were taken from Srikulnath et al. (2009b), those for A. carolinensis from Alföldi et al. (2011), those for E. quadrivirgata from Matsubara et al. (2006, 2012), and those for V. salvator macromaculatus from Srikulnath et al. (2013) 

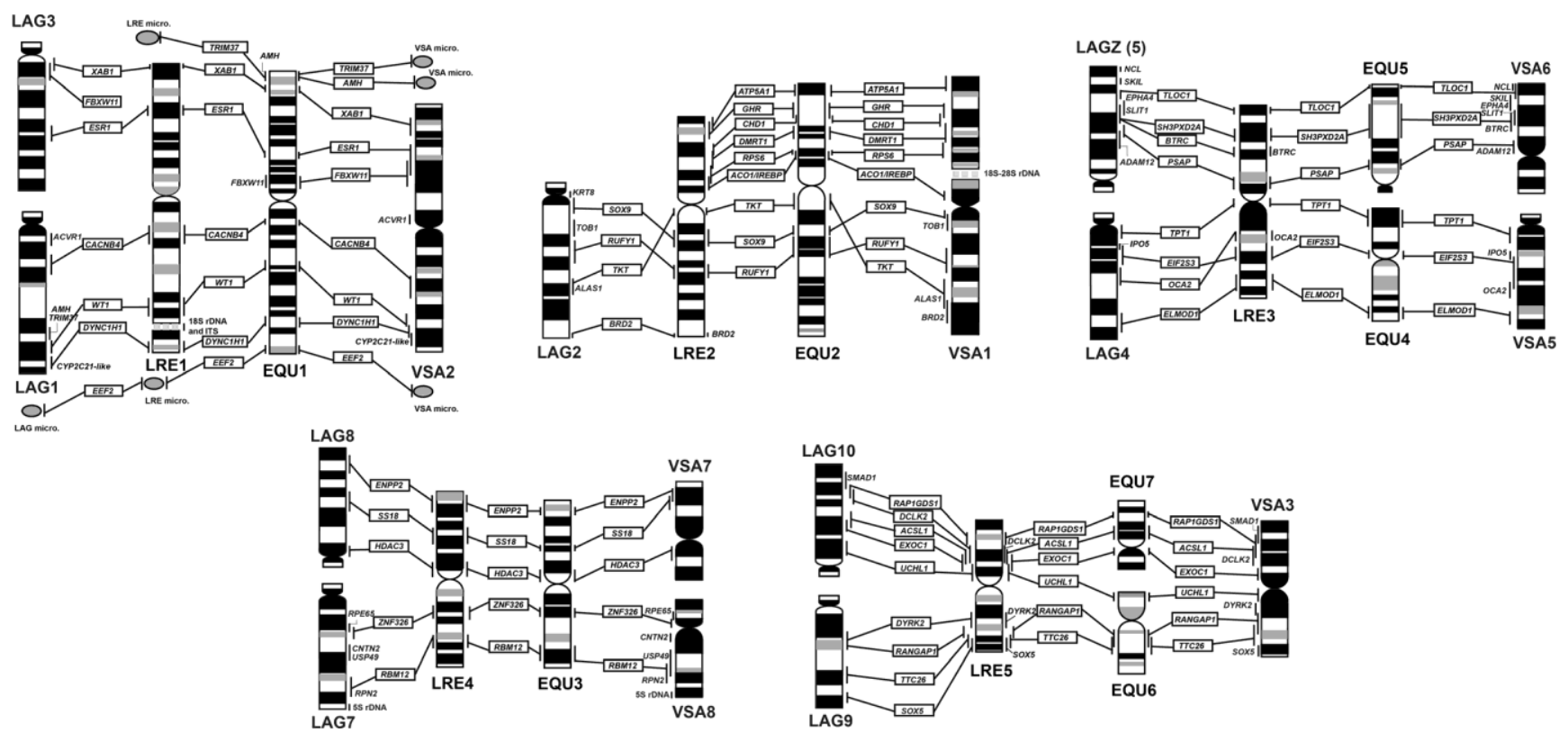

Fig. 6 Comparative cytogenetic maps of macrochromosomes among L. agilis, $V$. salvator macromaculatus, $L$. reevesii rubritaeniata, and E. quadrivirgata were constructed with 58 functional genes. The chro- mosome map of L. reevesii rubritaeniata (LRE) was taken from Srikulnath et al. (2009b). The ideogram of E. quadrivirgata (EQU) macrochromosomes was taken from Matsuda et al. (2005) and chromosomal locations of the genes in E. quadrivirgata from Matsubara et al. (2006,2012). The chromosome map of V. salvator macromaculatus was taken from Srikulnath et al. (2013). L. agilis chromosome (LAG)8, LAG10, LAG5(Z), VSA3, VSA6, VSA7, EQU5, and EQU7 are inverted to facilitate comparison. 

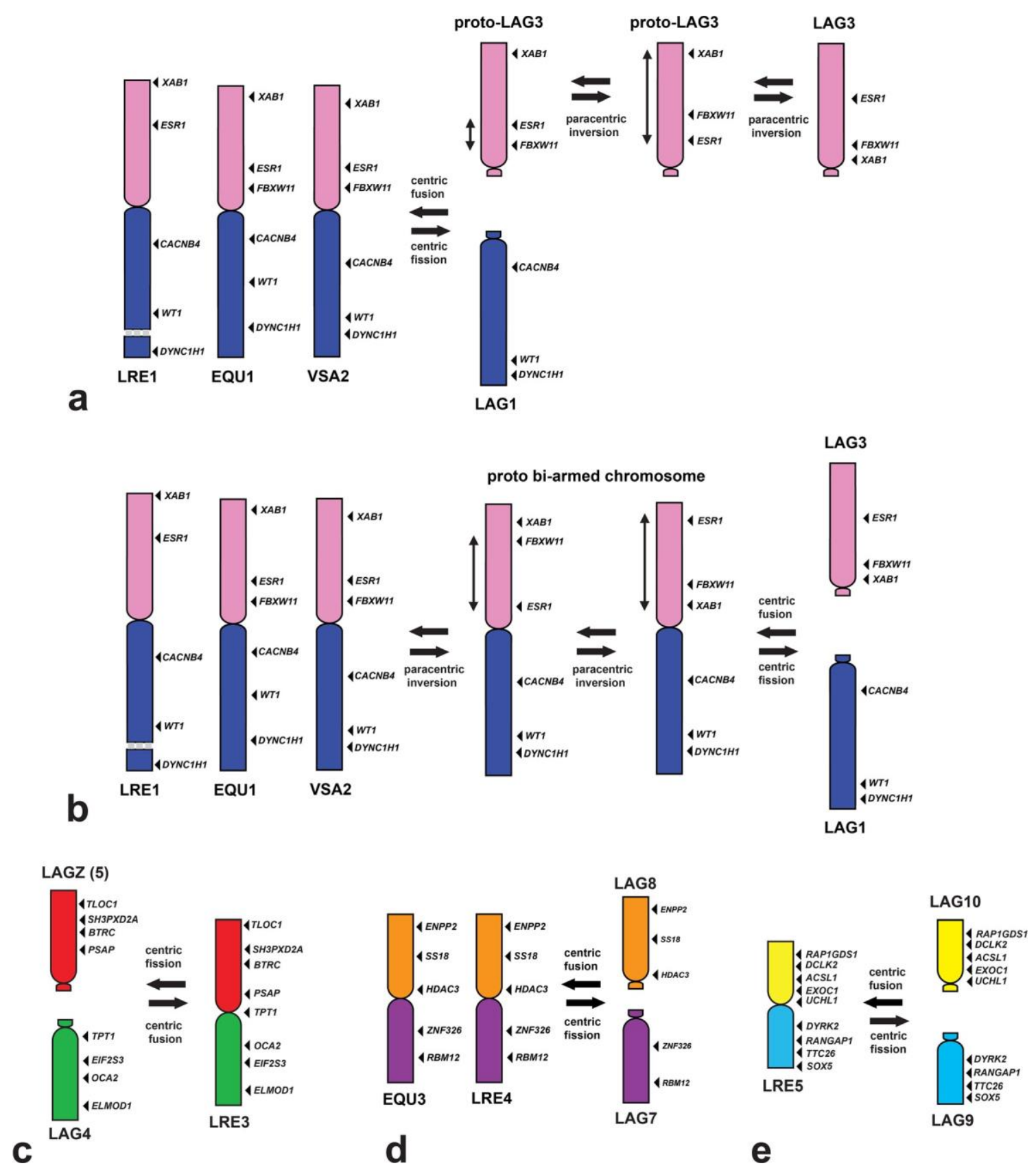

Fig. 7 Schematic representation of the process of chromosomal rear- rangements that occurred in L. agilis chromosomes 1, 3, 4, 5(Z), 7, 8, 9, and 10 [LAG1, LAG3, LAG4, LAGZ(5), LAG7, LAG8, LAG9, and

LAG10], V. salvator macromaculatus chromosome 2 (VSA2), L. reevesii rubritaeniata chromosomes 1, 3, 4, and 5 (LRE1, LRE3, LRE4, and LRE5), and E. quadrivirgata chromosomes 1 and 3 (EQU1 and EQU3). LAG3, LAGZ, LAG8, and LAG10 are inverted to facilitate comparison. Chromosomal locations of the genes are shown to the right of the chromosomes by arrowheads. Homologous chromosomes and/or chromosome segments are shown in the same color. The diagram schemati- cally summarizes the occurrences of LRE1, EQU1, VSA2, LAG1, and LAG3 (a, b); LRE3, LAG4, and LAGZ (c); LRE4, EQU3, LAG7, and LAG8 (d); and LRE5, LAG9, and LAG10 (e). Arrows indicate the directions of chromosomal rearrangement. Bidirectional arrows indicate the regions where paracentric inversions may have occurred

Acknowledgments This work was financially supported by Grants-in- Aid for Scientific Research on Innovative Areas (No. 23113004) and Scientific Research (B) (No. 22370081) from the Ministry of Education, Culture, Sports, Science and Technology, Japan. Mats Olsson thanks the Australian Research Council for financial support. 


\section{References}

Alföldi J, Di Palma F, Grabherr M et al (2011) The genome of the green anole lizard and a comparative analysis with birds and mammals. Nature 477:587-591. doi:10.1038/nature10390

Ciofi C, Swingland IR (1997) Environmental sex determination in rep- tiles. Appl Anim Behav Sci 51:251-265

Cree A, Thompson MB, Daugherty CH (1995) Tuatara sex determina- tion. Nature 375:543. doi:10.1038/375543a0

De Smet WHO (1981) Description of the orcein stained karyotypes of 27 lizard species (Lacertilia, Reptilia) belonging to the families Teiidae, Scincidae, Lacertidae, Cordylidae and Varanidae(Autarchoglossa). Acta Zool Pathol Antverpiensia 76:73-118

Ezaz T, Sarre SD, O’Meally D, Marshall Graves JA, Georges A (2010) Sex chromosome evolution in lizards: independent origins and rapid transitions. Cytogenet Genome Res 127:249-260. doi:10.1159/ 000300507

Ezaz T, Azad B, O'Meally Det al (2013) Sequence and gene content of a large fragment of a lizard sex chromosome and evaluation of can- didate sex differentiating gene R-spondin 1. BMC Genomics 14: 899. doi:10.1186/1471-2164-14-899

Gamble T, Geneva AJ, Glor RE, Zarkower D (2013) Anolis sex chromo- somes are derived from a single ancestral pair. Evolution. doi:10. 1111/evo.12328

Head G, May RM, Pendleton L (1987) Environmental determination of sex in the reptiles. Nature 329:198-199. doi:10.1038/329198a0 International

Chicken Genome Sequencing Consortium (ICGSC) (2004)

Sequence and comparative analysis of the chicken genome provide unique perspectives on vertebrate evolution. Nature 432:695-716. doi:10.1038/nature03154

Kawagoshi T, Uno Y, Matsubara K, Matsuda Y,Nishida C (2009) The ZW micro-sex chromosomes of the Chinese soft-shelled turtle (Pelodiscus sinensis, Trionychidae, Testudines) have the same ori- gin as chicken chromosome 15. Cytogenet Genome Res 125:125- 131. doi:10.1159/000227837

Kawagoshi T, Nishida C, Matsuda Y (2012) The origin and differentia- tion process of X and Y chromosomes of the black marsh turtle (Siebenrockiella crassicollis, Geoemydidae, Testudines). Chromosome Res 20:95-110. doi:10.1007/s10577-011-9267-7

Kawai A, Ishijima J, Nishida C, Kosaka A, Ota H, Kohno S, Matsuda Y (2009) The ZW sex chromosomes of Gekko hokouensis (Gekkonidae, Squamata) represent highly conserved homology with those of avian species. Chromosoma 118:43-51. doi:10.1007/ s00412-008-0176-2

Lang JW, Andrews HV (1994) Temperature-dependentsex determination in crocodilians. J Exp Zool 270:28-44. doi:10.1002/jez. 1402700105

Leaché AD, Sites JW Jr (2009) Chromosome evolution and diversifica- tion in north American spiny lizards (Genus Sceloporus). Cytogenet Genome Res 127:166-181. doi:10.1159/000293285

Matsubara K, Tarui H, Toriba Met al (2006) Evidence for different origin of sex chromosomes in snakes, birds, and mammals and step-wise differentiation of snake sex chromosomes. Proc Natl Acad Sci U S A 103:18190-18195. doi:10.1073/pnas.0605274103

Matsubara K, Kuraku S, Tarui H et al (2012) Intra-genomic GC hetero- geneity in sauropsids: evolutionary insights from cDNA mapping and GC 3 profiling in snake. BMC Genomics 13:604. doi:10.1186/ 1471-2164-13-604

Matsuda Y,Chapman VM (1995) Application of fluorescence in situ hybridization in genome analysis of the mouse. Electrophoresis 16: 261-272

Matsuda Y, Nishida-Umehara C, Tarui H et al (2005) Highly conserved linkage homology between birds and turtles: bird and turtle chromosomes are precise counterparts of each other. Chromosome Res 13:601-615. doi:10.1007/s10577-005-0986-5

Nishida-Umehara C, Tsuda Y, Ishijima J, Ando J, Fujiwara A et al (2007) The molecular basis of chromosome orthologies and sex chromo- somal differentiationin palaeognathousbirds. Chromosome Res 15: 721-734. doi:10.1007/s10577-007-1157-7

Odierna G, Olmo E, Cobror O (1987) Taxonomic implications of NOR- localization in lacertid lizards. Amphibia-Reptilia 87:373-382

Odierna G, Kupriyanova L, Capriglione T, Olmo E (1993) Further data on sex chromosomes of Lacertidae and a hypothesis on their evolu- tionary trend. Amphibia-Reptilia 14:1-11

Odierna G, Olmo E, Caputo V, Capriglione T, in den Bosch HAJ (1995) Karyological affinity between Lacerta fraasii Lehrs, 1910 and Lacerta parva Boulenger, 1887. Amphibia-Reptilia 16:293-297

Olmo E, Signorino G (2005) Chromorep: a reptile chromosomes data- base. Internetreferences. Retrieved from: http://chromorep.univpm. it. $6 / 04 / 2013$

Olmo E, Odierna G, Capriglione T (1987) Evolution of sex- chromosomes in lacertid lizards. Chromosoma 96:33-38

Olmo E, Odierna G, Capriglione T, Caputo V (1991) A karyological approach to the systematic of Lacertidae (Reptilia, Sauria). Rev Esp Herpetol 6:81-90

Pokorná M, KratochvílL, KejnovskýE(2011)Microsatellite distribution on sex chromosomes at different stages of heteromorphism and heterochromatinization in two lizard species (Squamata: Eublepharidae: Coleonyx elegans and Lacertidae: Eremias velox). BMC Genet 12:90. doi:10.1186/1471-2156-12-90

Pyron RA, Burbrink FT, Wiens JJ (2013) A phylogeny and revised classification of Squamata, including 4161 species of lizards and snakes. BMC Evol Biol 13:93. doi:10.1186/1471-2148-13-93

Shedlock AM, Edwards SV (2009) Amniota. In: Hedges SB, Kumar S (eds) The timetree of life. Oxford University Press, New York, pp 375-379

Srikulnath K, Matsubara K, Uno Yet al (2009a) Karyological character- ization of the butterfly lizard (Leiolepis reevesii rubritaeniata, Agamidae, Squamata) by molecular cytogenetic approach. Cytogenet Genome Res 125:213-223.doi:10.1159/000230005

Srikulnath K, Nishida C, Matsubara Ketal (2009b) Karyotypic evolution in squamate reptiles: comparative gene mapping revealed highly conserved linkage homology between the butterfly lizard (Leiolepis reevesii rubritaeniata, Agamidae, Lacertilia) and the Japanese four-striped rat snake (Elaphe quadrivirgata, Colubridae, Serpentes). Chromosome Res 17:975-986. doi:10.1007/s10577- 009-9101-7

Srikulnath K, Uno Y, Nishida C, Matsuda Y (2013) Karyotype evolution in monitor lizards: cross-species chromosome mapping of cDNA reveals highly conserved synteny and gene order in the Toxicofera clade. Chromosome Res 21:805-819. doi:10.1007/s10577-013-9398-0

Uetz P (2014) The TIGR reptile database. The EMBL reptile database. Retrieved from: http://www.reptile-database.org/

Uno Y, Nishida C, Tarui H et al (2012) Inference of the protokaryotypes of amniotes and tetrapods and the evolutionary processes of microchromosomes from comparative gene mapping. PLoS ONE 7:e53027. doi:10.1371/journal.pone.0053027

Vidal N, Hedges SB (2005) The phylogeny of squamate reptiles (lizards, snakes, and amphisbaenians) inferred from nine nuclear protein-coding genes. C R Biol 328:1000-1008. doi:10.1016/j.crvi.2005.10.001 